Heredity (1973), 30 (1), 27-32

\title{
EFFECTS OF DISRUPTIVE SELECTION
}

\section{SELECTIVE MIGRATION}

\author{
JOHN B. GIBSON and J. M. THODAY \\ Department of Genetics, University of Cambridge, Cambridge CB4 $1 \mathrm{XH}$
}

Received 28.ii.72

\begin{abstract}
SUMMARY
Two populations of Drosophila melanogaster were exposed to disruptive selection for sternopleural bristle number. There was 25 per cent. gene flow between the halves of the populations selected for high bristle number and the halves selected for low bristle number. In one population the migrant males were selected in the same direction as the flies in the recipient half $\left(D^{+}\right)$and in the other population the migrants were selected in the opposite direction to the flies in the recipient half $\left(D^{-}\right)$. Both populations rapidly showed divergence between their halves and for the first three generations the divergences were very similar. The $D^{+}$population eventually attained a greater divergence than the $D^{-}$population. The $D^{-}$population maintained a cryptic sex-linked polymorphism. The results are compared to those of Millicent and Thoday (1961) and discussed in relation to the effects of selective migration and disruptive selection. It is concluded that divergence under sufficiently intense disruptive selection is possible even when the migration is much less favourable to divergence than it would be under random mating.
\end{abstract}

\section{INTRODUGTION}

Millicent AND Thoday $(1960,1961)$ showed that polymorphism could be established in a population of Drosophila melanogaster exposed to disruptive selection with 25 per cent. gene flow.

In their experiments half of the population was selected for high and half for low sternopleural bristle number. Only male flies migrated between the two halves of the population. The migrants from the low half into the high half were selected for high bristle number and migrants in the other direction were selected for low bristle number. They referred to this as positive assortative mating $\left(D^{+}\right)$.

Most of the divergence in bristle number in one of their 25 per cent. gene flow lines was maintained when the direction in which the migrants were selected was reversed, so that migrants were selected for bristle number in the same, instead of the opposite, direction to that occurring in the donor half of the population. They referred to this as negative assortative mating $\left(D^{-}\right)$. The question remained whether a system of selective migration as used in their $D^{-}$line could also give rise to divergence, as distinct from maintaining a difference already established. The present experiments were carried out in an attempt to answer this question and to compare the effects on divergence of the two selective migration systems in samples of the same base population.

\section{MAterials AND MEthods}

The experimental populations were initiated with a Drosophila melanogaster wild stock (Athens), derived from a single female and maintained at $25^{\circ} \mathrm{C}$. in mass cultures for four generations prior to the experiment. 
The methods of maintenance of the lines and the mating systems were the same as those used by Millicent and Thoday (1961) for their 25 per cent. gene flow lines. However, as the description of the $D^{-}$negative assortative mating breeding programme on page 215 of their paper is misleading, the mating systems used in the present experiments are shown in table 1.

TABLE 1

Mating systems

25 per cent. gene flow with positively selective migration

$\left(D^{+}\right)$

25 per cent. gene flow with negatively selective migration

$\left(D^{-}\right)$

Cultu
A
B
C
D

$\begin{array}{ll}\text { A } & \text { AH } \\ \text { B } & \text { BH } \\ \text { C } & \text { CL } \\ \text { D } & \text { DL }\end{array}$

$q$
parent
AH
BH
CL
DL

\begin{tabular}{|c|c|c|c|}
\hline \multicolumn{4}{|c|}{$\delta^{t}$ parent in generation } \\
\hline 1 & 2 & 3 & 4 \\
\hline $\mathrm{BH}$ & $\mathrm{DH}$ & $\mathrm{BH}$ & $\mathrm{DH}$ \\
\hline $\mathrm{CH}$ & $\mathrm{AH}$ & $\mathrm{CH}$ & $\mathrm{AH}$ \\
\hline DL & $\mathrm{BL}$ & DL & $\mathrm{BL}$ \\
\hline$\overline{\mathrm{AL}}$ & $\mathrm{CL}$ & $\mathrm{AL}$ & $\mathrm{CL}$ \\
\hline
\end{tabular}

$\mathrm{H}$ indicates the highest and $\mathrm{L}$ the lowest fly chosen from a sample of 20 . Only males migrate. ABCD indicate which culture the fly came from.

The terms positive assortative mating and negative assortative mating used by Millicent and Thoday for the two systems of migration can be confusing and the phrases positively selective migration and negatively selective migration will be used instead. The respective symbols $D^{+}$and $D^{-}$are retained.

Each population was represented by four single-pair cultures per generation. Twenty flies of each sex from each culture were assayed for sternopleural chaeta number. Three insurance cultures were set up for each mating. The proportion of flies selected ( 1 in 20) was the same as that used in the experiments described in the first four of this series of papers.

The experiment was terminated after nine generations of selection because the general conclusions were by then clear, and there was pressure on our facilities for handling flies.

\section{RESULTS}

The results are presented in a comparable form to those of Millicent and Thoday (1961). The mean bristle numbers of each culture in each generation are shown in fig. 1 and the rates of divergence in bristle number between the two halves of the populations are illustrated in fig. 2 .

The divergence obtained in the 25 per cent. gene flow line with positively selective migration $\left(D^{+}\right)$was immediate and more rapid than that in the comparable line of Millicent and Thoday. In their line the difference between the two halves of the population was 5.5 bristles per fly after 25 generations of selection. The present line showed a difference of 7 bristles per fly by $\mathrm{S} 9$ when the line was terminated.

Surprisingly, the line maintained with negatively selective migration $\left(D^{-}\right)$also gave rise to immediate divergence and for the first three genera- 


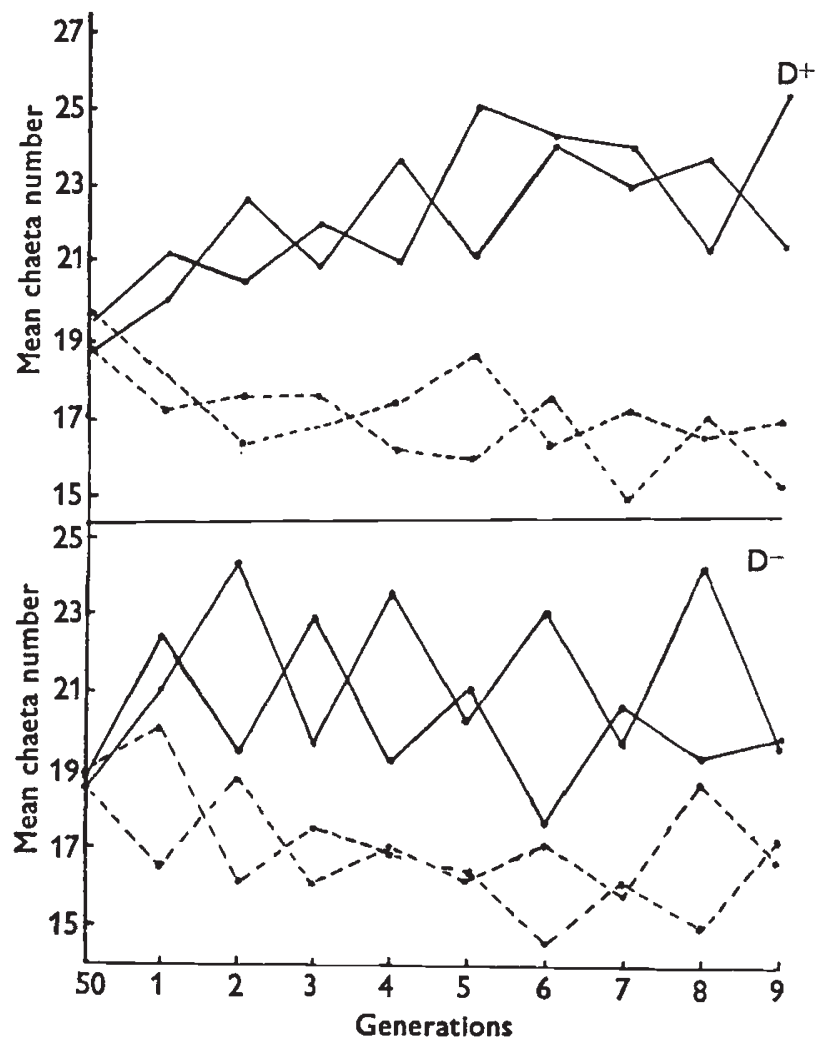

Frg. 1.-Mean bristle numbers for the four female sub-lines of $D^{+}$(upper figure) and $D^{-}$ (lower figure). Solid lines high cultures, dotted lines low cultures.

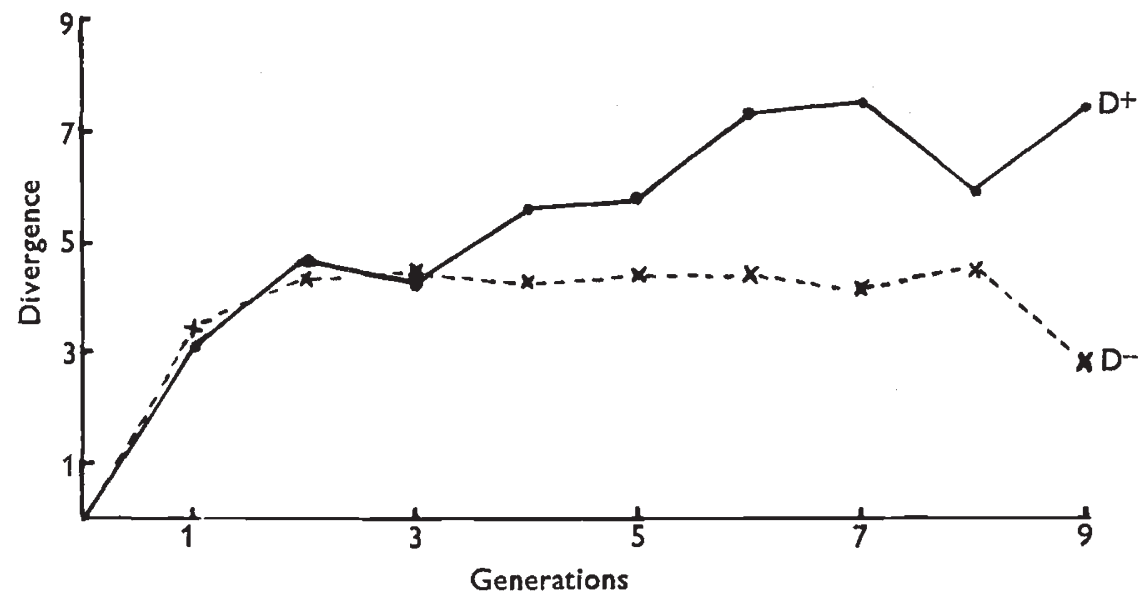

FIG. 2.-Mean divergence in bristle number per fly. Solid line $D^{+}$, dotted line $D^{-}$.

tions of selection the two lines behaved in a similar way. Thereafter the $D^{+}$line continued to diverge but the $D^{-}$line maintained, rather precisely, a difference of 3 bristles per fly until S9 where it fell to 2 bristles per fly. 
As in the lines of Millicent and Thoday the two cultures in the high half of the population (and also the two in the low half) alternated in extreme mean bristle number each generation (fig. 1). This alternation is more pronounced in the $D^{-}$line which is not unexpected as, of the two selected high females in a particular generation of the $D^{- \text {-line, one is mated }}$ to a high male from the high half and the other to a low male selected from the low half of the population. Nevertheless, the $D^{-}$line maintained a divergence of 3 bristles per fly for five generations of selection.

The distributions of bristle numbers in the base population and at S9 in both lines (fig. 3) illustrate the increases in phenotypic variance brought about by disruptive selection under these systems of migration.

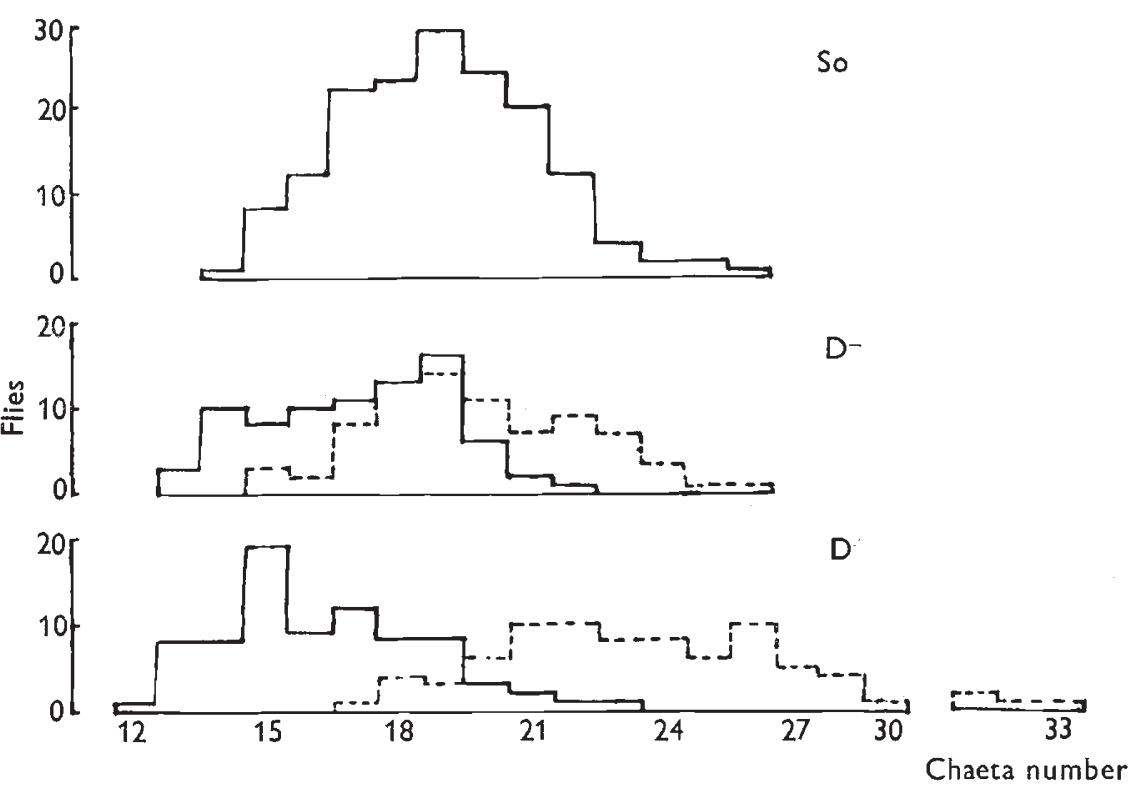

FIg. 3.-Distributions of bristle numbers in the base population and the $D^{+}$and $D^{-}$populations. Solid lines high culture, dotted lines low cultures.

\section{Discussion}

These results provide further evidence for the effectiveness of disruptive selection (Mather, 1955; Thoday and Boam, 1959). In particular they demonstrate that divergence between two halves of a population subjected to disruptive selection is possible with 25 per cent. gene flow even when the migrants are selected in the direction opposite to that applied in the recipient half of the population, that is when migration is negatively selective.

The results for the $D^{+}$line are consonant with those of Millicent and Thoday (1961) except that the divergence obtained was larger. This difference presumably arises from greater genetic variance in the base population. Millicent and Thoday in fact had evidence that the "Dronfield" stock they used was less responsive than it had been in previous disruptive selection experiments (Thoday, 1959), whereas the present experiments utilised a newly captured wild population. 
That the present $D^{+}$line was not so clearly polymorphic after nine generations of selection as Millicent and Thoday's line after 25 generations is hardly surprising. What is perhaps surprising is that the present $D^{+}$ and $D^{-}$lines showed the same rate of divergence over the first three generations of selection.

One other feature of the results for the $D^{-}$line, requires explanation. In each generation, the hybridised culture in the high half of the $D^{-}$population has a higher mean bristle number than the hybridised culture in the

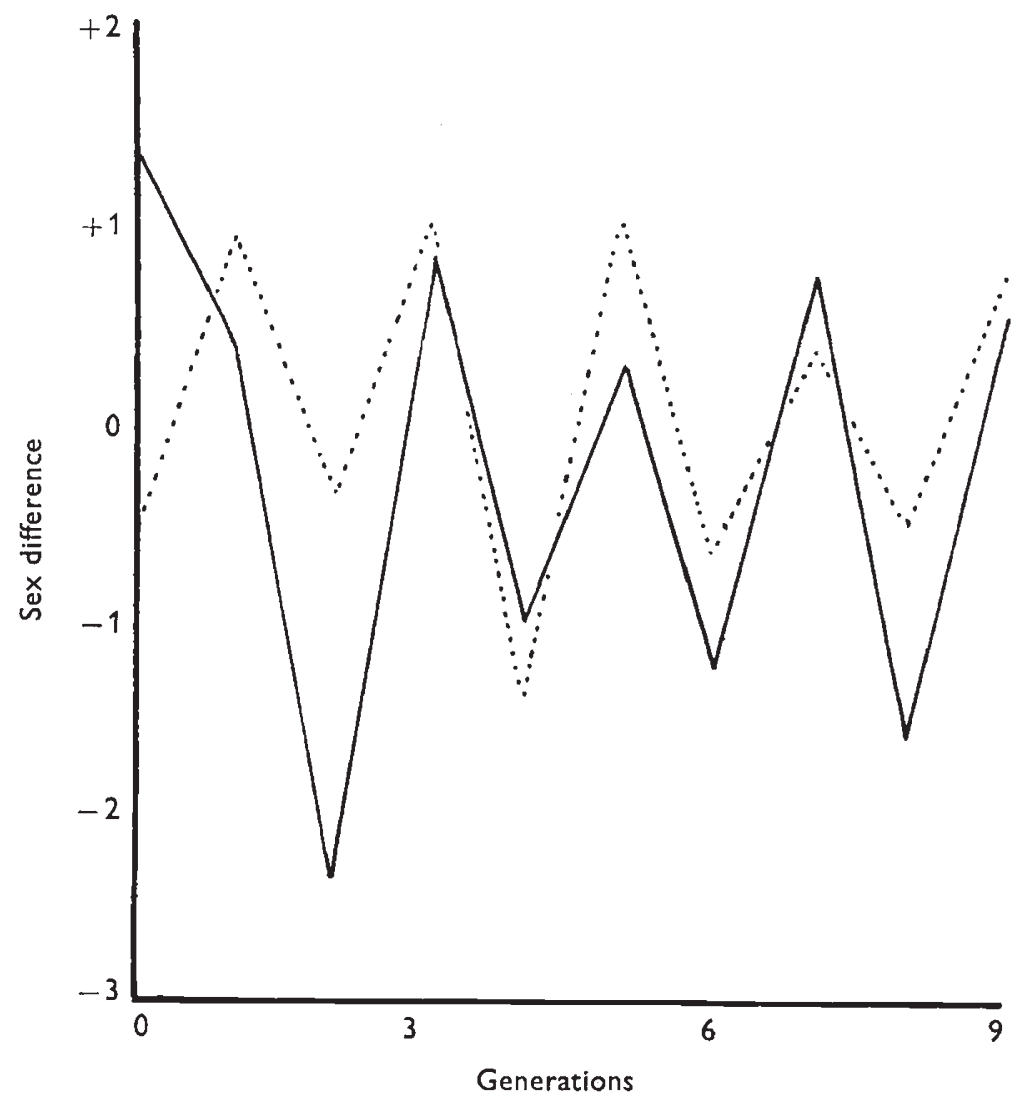

FIG. 4.-Differences between the sex differences in each of the two cultures in each half of the $D^{-}$population. Solid line $\left(\mathrm{A} q-\mathrm{A}_{\delta^{\top}}\right)-\left(\mathrm{B} q-\mathrm{B}^{\top}\right)$, dotted line $\left(\mathrm{D} q-\mathrm{D}_{\delta^{\top}}\right)-\left(\mathrm{C} q-\mathrm{C}^{\top}\right)$.

low half of the population. It is difficult to see any explanation for this in terms of segregating autosomal genes, but the difference could be explained if there were cytoplasmic or maternal inheritance or if sex-linked genes were segregating. The latter hypothesis accounts for the result.

If we postulate a single sex-linked locus affecting bristle number, with + and - alleles equal and opposite in effect, then $\mathrm{H} q \times \mathrm{L} \sigma$ matings in the high half of the population could be represented as $\frac{t}{+}+x=\delta$. Such matings would give rise to high bristle number males and intermediate bristle number females. In the next generation the $\mathrm{H}$ o $\times \mathrm{H}$ o mating, i.e. 
$\pm q \times \pm \Xi$, would give rise to equal numbers of high and intermediate bristle number females, together with equal numbers of high and low bristle number males. In the low half of the population the $\mathrm{L}+\mathrm{q} \times \mathrm{H}$ व mating, i.e. 二 $q \times \pm \delta$, would produce intermediate bristle number females and low

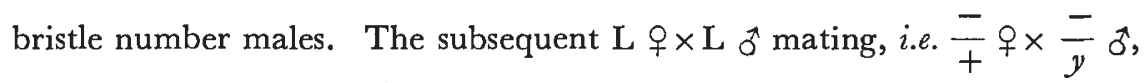
would generate low and intermediate bristle number females together with low and high bristle number males. The consequences would be similar if more loci were involved.

On this hypothesis the sex difference in bristle number in the two cultures of either half of the population would be expected to alternate in alternate generations. The differences between the sex difference of the two cultures in each half of the $D^{-}$population (fig. 4) are in good agreement with this hypothesis. Thus disruptive selection with 25 per cent. gene flow and negatively selective migration in this experiment, established a cryptic sex-linked polymorphism.

Migration between parts of populations can be of three general kinds, two of which involve selection. The migrants could be a random sample of the donor population and Streams and Pimental (1961) provided evidence that such migration did not prevent divergence and could even enhance the responsiveness of a population to selection. The two extreme forms of selective migration are represented in the two mating systems used in the present experiments (though migration need not be restricted to one sex). The $D^{-}$system is the most unfavourable to divergence that we can devise. The results reinforce the conclusion that disruptive selection can promote divergence between parts of a population and show this to be possible when selective migration is deliberately made unfavourable to such divergence.

\section{REFERENGES}

MATHER, K. 1955. Polymorphism as an outcome of disruptive selection. Evolution, 9, 52-61. MILliCENT, E., AND THODAY, J. M. 1960. Gene flow and divergence under disruptive selection. Science, 131, 1311-1312.

Millicent, E., AND thoDAY, J. M. 1961. Effects of disruptive selection. IV. Gene flow and divergence. Heredity, 15, 119-217.

STREAMs, F. A., AND PIMENTAL, D. 1961. Effects of immigration on the evolution of populations. Amer. Nat., 95, 201-209.

thoday, J. M., AND Boam, т. в. 1959. Effects of disruptive selection. II. Polymorphism and divergence without isolation. Heredity, 13, 205-218. 